\title{
Kidney-paired donation: do transplantations need to be performed simultaneously?
}

K idney-paired donation-where patients who have incompatible but willing living donors receive transplants from other living donors (nondirected donors or donors from other incompatible pairs) — is increasing in the USA. A study recently published in the American Journal of Transplantation reports that performing these transplantation chains nonsimultaneously increases the number of transplants carried out.

Controversy surrounds the issue of whether transplantation chains should be performed simultaneously or nonsimultaneously. Until quite recently, all transplantations in a chain were performed at the same time (dominopaired donation [DPD] chains), but nonsimultaneous chains initiated by nondirected donors (nonsimultaneous extended altruistic donor [NEAD] chains) are becoming more common. "Some advantages of nonsimultaneous chains include the fact that chains can become longer because they are less constricted by logistical factors, and that more people can be transplanted; in addition, the lack of need for reciprocity allows donors to be used more efficiently so that quality can be optimized," notes Michael Rees, corresponding author on the latest paper. "The disadvantage is obviously that people trusted at the end of chains-referred to as bridge donors-might renege and not 'pay forward' the gift given to their loved one."

"Although nonsimultaneous chains are fast becoming fairly standard, a paper published in 2009 concludedthrough the use of simulations-that nonsimultaneous chains wouldn't produce more transplants than simultaneous chains," says Alvin Roth, another author on the recent study. "Given our data in the real world, we disagreed with the data and assumptions from this paper," adds Rees. "They assumed that chains would have to be limited to a length of three during any

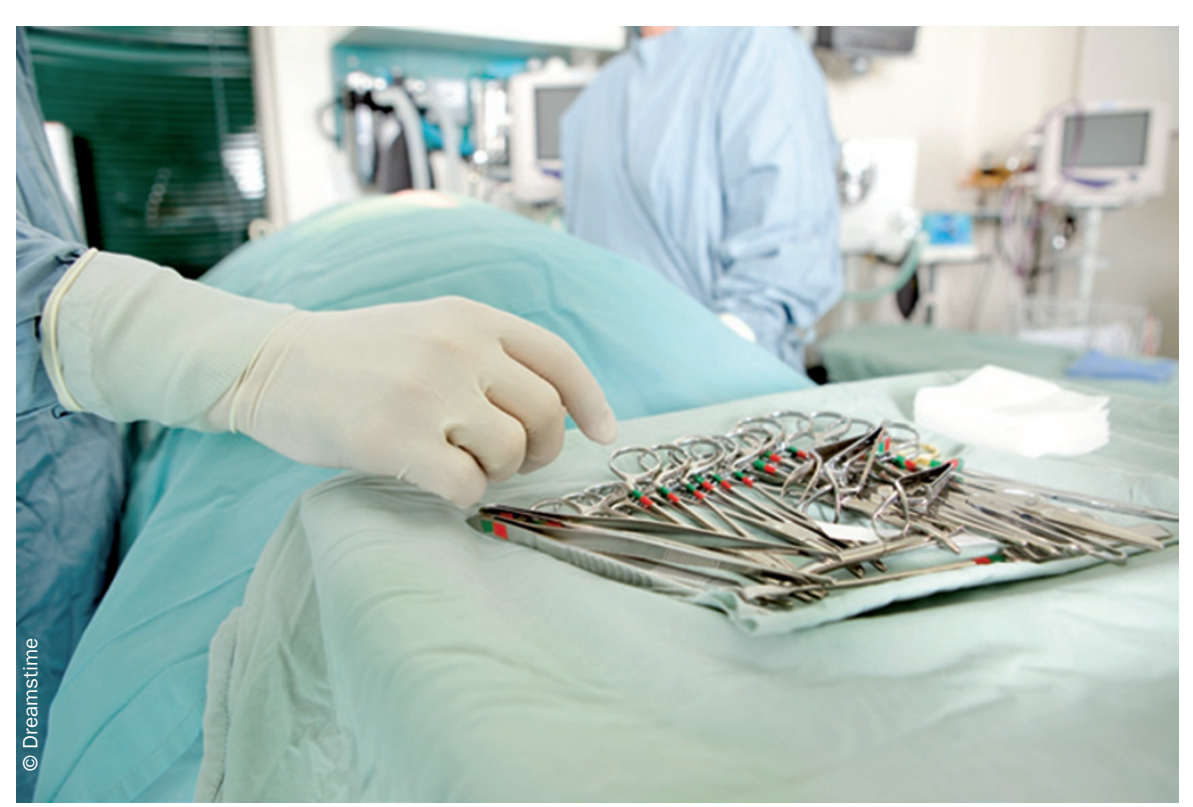

computerized attempt to identify chains. We felt that longer chains had to be part of the assumptions."

Rees' team used empirical data from the registry of the Alliance for Paired Donation to build a simulation model to test whether simultaneous DPD chains or NEAD chains would result in more transplants being performed. When they used the same assumptions as the 2009 paper, they confirmed the 2009 findings. However, when they allowed NEAD chains to be longer than three pairs, as currently often occurs in clinical practice, they found that NEAD chains produced more transplants than DPD chains.

The authors also investigated the percentages of highly sensitized patients (panel-reactive antibody level $>80 \%$ ) and patients with blood type $\mathrm{O}$ who would receive transplants with the different methods. They found that increased numbers of these hard-to-transplant groups of patients received a transplant when NEAD chains were used. However, the authors do note that in clinical practice it will be important to limit the length of time that bridge donors are allowed to wait before a decision is made to end a chain by donating the bridge donor's kidney to a candidate on the deceased donor waiting list who lacks a willing donor.

"Going forward, one of the big questions that we hope to explore is how best to end nonsimultaneous chains," states Roth. "That is, when does it make sense to ask the final donor to wait for a future transplant opportunity, and when does it make sense for them to donate to someone on the waiting list? Sometimes a bridge donor can facilitate more and/or better transplants than would an immediate transplant to a waiting list candidate, but this may not always be the case. Our paper included both types of endings of NEAD chains, but we haven't yet studied when each kind of ending would be most useful."

Rebecca Ireland

Original article Ashlagi, l. et al. Nonsimultaneous chains and dominos in kidney-paired donation-revisited. Am. J. Transplant. 11, 984-994 (2011) 